\title{
THz signal transmission in a compact modular waveguide system
}

\author{
A. von Bieren ${ }^{a}$, E. de Rijk ${ }^{a}$, A. Macor ${ }^{a}$, J.-Ph. Ansermet ${ }^{b}$, B. Maffei ${ }^{c}$, G. Pisano ${ }^{c}$ \\ ${ }^{a}$ SWISSto12 SA, EPFL Innovation Park, 1015 Lausanne, Switzerland \\ ${ }^{b}$ Institute of Condensed Matter Physics, EPFL, 1015 Lausanne, Switzerland \\ ${ }^{c} J B C A$, School of Physics and Astronomy, The University of Manchester, Manchester M13 9PL, UK
}

\begin{abstract}
The transmission properties of a compact circular corrugated waveguide system are investigated experimentally in the WM-380 band from 500 to $750 \mathrm{GHz}$. Up to $700 \mathrm{GHz}$, the average transmission losses range from below the detection limit to $3.4 \mathrm{~dB} / \mathrm{m}$, i.e. more than four orders of magnitude lower than rectangular waveguides. The propagation of the lowest-loss $\mathbf{H E}_{11}$ mode is found to be dominant, and long-term measurements of phase and amplitude demonstrate high transmission stability.

Index Terms - Circular corrugated waveguides, converters, miter bends, sub-millimeter, terahertz, signal transmission.
\end{abstract}

\section{INTRODUCTION}

Terahertz (THz) technology is becoming an increasingly active field of research and development, with a vast number of applications ranging from medical devices to communication systems. Rectangular waveguides are widely used as the transmission medium in the sub-THz range. When approaching $1 \mathrm{THz}$, however, such waveguides suffer from increased losses and become prohibitively difficult to manufacture due to tight fabrication tolerances. For instance an ideal Au-coated WM-380 waveguide $(500-750 \mathrm{GHz}$, also called WR-1.5) exhibits losses of at least $47 \mathrm{~dB} / \mathrm{m}$, not taking into account roughness-induced losses [1]. This drastically reduces the accessible length and dynamic range in test and measurement applications.

Overmoded circular corrugated waveguides (CCWs) are often employed to transmit high-power microwave signals in plasma physics experiments. Such waveguides usually propagate hybrid electric (HE) modes. In particular the fundamental $\mathrm{HE}_{11}$ mode is known for its exceptionally low losses across large bandwidths $(<0.05 \mathrm{~dB} / \mathrm{m}$ for the abovementioned example) [2]. Previously, $\mathrm{CCWs}$ were limited to lower frequencies due to the precision of traditional manufacturing technologies. Thanks to the introduction of the 'stacked rings' technology developed by SWISSto12 [3], the frequency range accessible by $\mathrm{CCWs}$ has recently been extended to the $\mathrm{THz}$ range. Such $\mathrm{CCW}$ s are therefore considered a viable alternative to rectangular waveguides for a wide range of $\mathrm{THz}$ applications.

In this paper we present transmission and stability measurements of a modular set of CCW components developed and manufactured by SWISSto12. The brass prototypes are designed for the WM-380 band from 500 to $750 \mathrm{GHz}$ [4]. The analyzed components are an optimized combination of converters, straight segments and bends based on the 'stacked rings' technology that enables broadband and low-loss operation. A high performance connection system between the components allows for modular path building, high transmission stability and efficient power coupling to solid-state devices.

\section{WAVEGUIDE MODULES}

The set comprises three main elements that can be combined individually (see Fig. 1(a)): (i) Mode converters (Fig. 1(c)) provide the interface between rectangular waveguides (UG-387/UM flange), such as solid-state sources and wafer probes, and the CCW components described below. The converters consist of a double-step transition followed by a corrugated Winston horn, providing efficient mode conversion from $\mathrm{TE}_{10}$ (the fundamental mode in rectangular waveguides) to $\mathrm{HE}_{11}$ with low cross-polarization levels [5]. (ii) Straight corrugated waveguide segments (Fig. 1(b)) with 8 $\mathrm{mm}$ inner diameter are suited for signal propagation over variable lengths. The corrugation employed has a pitch of 0.25 $\mathrm{mm}$, a slot thickness of $0.05 \mathrm{~mm}$ and a depth of $0.145 \mathrm{~mm}$. (iii) Right angle bends (Fig. 1(d)) are compact miter bends that allow building custom waveguide paths (see Fig. 1(a)) as

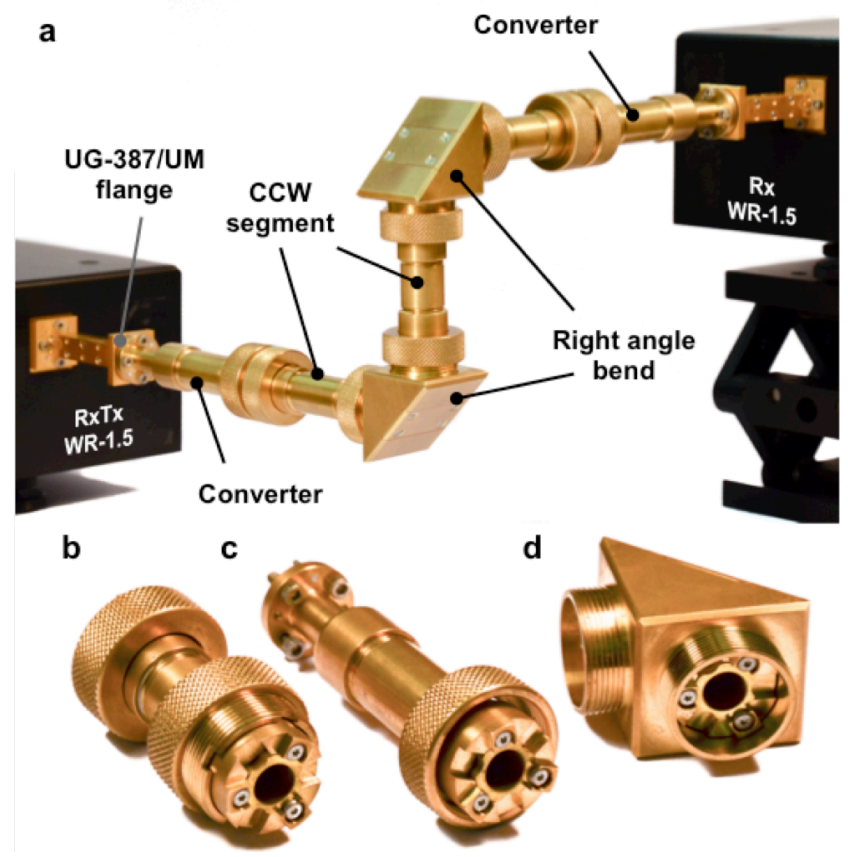

Fig. 1. (a) Assembled waveguide system connecting two VNA frequency extenders over a total distance of about $30 \mathrm{~cm}$. Components of the modular waveguide: (b) $55 \mathrm{~mm}$ circular corrugated waveguide module, (c) Converter, (d) Right angle bend. 
required for example in cryogenic probe stations. All components are equipped with self-aligning connection flanges for quick and reliable (dis-)connection.

The prototype components analyzed here have been designed for the WM-380 waveguide band. However, the used manufacturing technology can be scaled to lower frequencies as well as up to $1 \mathrm{THz}$ and beyond with only minor modifications while maintaining a similar performance.

\section{RESULTS AND DISCUSSION}

All measurements shown are performed using an Agilent N5224A Vector Network Analyzer equipped with frequency extender modules from VDI for 500 to $750 \mathrm{GHz}$.

\section{A. $H E_{11}$ Mode Content of the Converter}

As the propagation of the $\mathrm{HE}_{11}$ mode is key to achieving low-loss transmission, first we verify the modal purity attained by the converter module. For this purpose, we carry out spatially resolved measurements of the electric field at the converter output by mounting one of the extender modules on a 3-axis translation stage. Using a short pick-up waveguide phase and relative amplitude of the electric field can be sampled at arbitrary points in space with a lateral resolution given by the waveguide aperture (381 x $191 \mu \mathrm{m}$ for WR-1.5).

$20 \times 20 \mathrm{~mm}$ scans with $0.5 \mathrm{~mm}$ resolution are taken at a distance of $20 \mathrm{~mm}$ from the aperture. The measured complex field is then numerically back-propagated by $20 \mathrm{~mm}$ [6]. Fig. 2(b) shows the resulting intensity distribution at the output aperture of the converter for three selected frequencies. As expected, the images show a Gaussian-like distribution reproducing the aperture's inner diameter of $8 \mathrm{~mm}$.

The projection of the obtained complex field distribution onto an ideal $\mathrm{HE}_{\mathrm{mn}}$ distribution yields the fraction of the total power carried by the respective mode [6]. Fig. 2(a) shows the
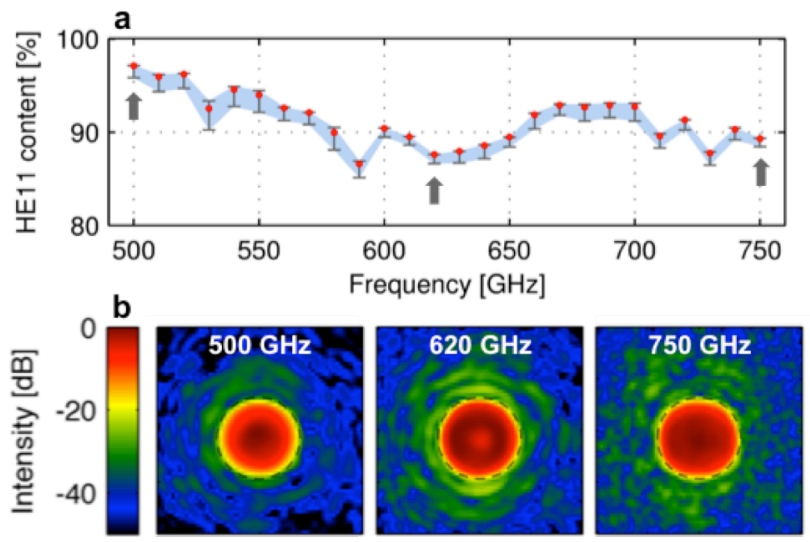

Fig. 2. (a) $\mathrm{HE}_{11}$ mode content generated by the corrugated horn converter between $500 \mathrm{GHz}$ and $750 \mathrm{GHz}$. Red dots show the mode content in \%, and error bars indicate the estimated error due to possible misalignment. The blue shaded region is a guide to the eye. (b) $20 \times 20 \mathrm{~mm}^{2}$ intensity distribution at the output aperture converter for $500 \mathrm{GHz}, 620 \mathrm{GHz}$, and $750 \mathrm{GHz}$. Dashed black circles indicate the position of the $8 \mathrm{~mm}$ diameter output aperture. resulting $\mathrm{HE}_{11}$ power content within the considered band. Error bars indicate the estimated maximum error due to misalignment between the converter and the measurement plane $(\leq 0.1 \mathrm{~mm}$ offset along two orthogonal in-plane axes and $\leq 3 \mathrm{~mm}$ along the propagation axis). Strikingly, the power content stays above $85 \%$ across the full band, confirming that wave propagation in the $\mathrm{CCW}$ system is dominated by the low-loss $\mathrm{HE}_{11}$ mode. Importantly, the $\mathrm{HE}_{11}$ power content stays above $85 \%$, even after propagation through four waveguide modules and two right angle bends (data not shown). The strongest contribution due to higher-order modes is observed around the band center and at the upper band edge. This non-Gaussian contribution can also be identified in the intensity images for 620 and $750 \mathrm{GHz}$ depicted in Fig. 2(b).

\section{B. Attenuation Losses in Straight Waveguide Modules}

S-parameter traces are calibrated using a TRL calibration between the test ports (UG-387/UM flanges) and acquired at 1 $\mathrm{kHz}$ IF bandwidth. The filtered traces shown are obtained by applying time-domain gating to the measurement data (window width $100 \mathrm{ps}$, centered around main path $\left(\mathrm{S}_{21}\right)$ or mirror position $\left(S_{11}\right)$ ) in order to remove spurious resonances.

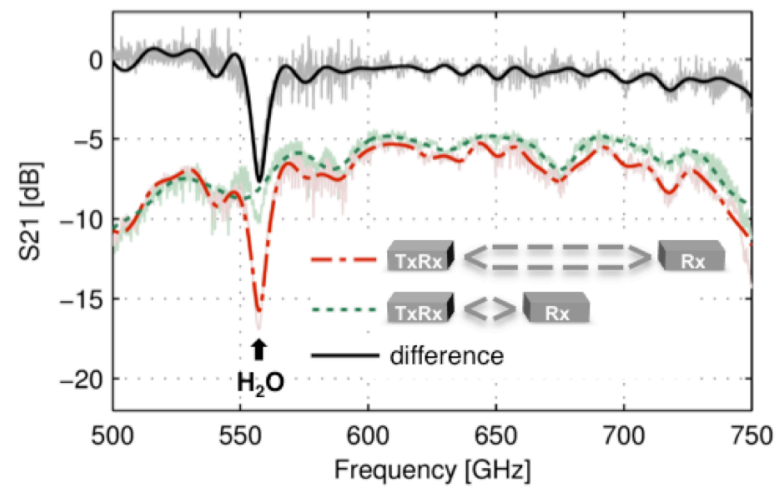

Fig. 3. $\mathrm{S}_{21}$ data for two transmission line configurations: converter to converter (dashed line), complete transmission line consisting of two converters connected by four $55 \mathrm{~mm}$ straight waveguide sections (dash-dotted line), difference between previous two curves illustrating losses due to a $220 \mathrm{~mm}$ waveguide only (continuous line). Data is filtered using time-domain gating and calibrated between input/output ports of frequency extenders. Light lines show raw data.

$\mathrm{S}_{21}$ measurements are performed for two configurations of the modular transmission line (see Fig. 3). First, we measure transmission through two converters connecting the test ports in a in a face-to-face configuration. This measurement serves as a reference that includes losses due to both, the interfaces with the test ports as well as the converters itself. Second, we add four identical $55 \mathrm{~mm}$ long CCW sections in between the two converters while leaving the test port connections unchanged. As shown in Fig. 3, the overall transmission behavior is dominated by the performance of the two converters with a total attenuation of 5 to $10 \mathrm{~dB}$. Our analysis shows that this can be attributed to mechanical inaccuracies of 
the rectangular waveguide interface of the prototypes studied here. As indicated in Fig. 3, the strong dip close to $560 \mathrm{GHz}$ is due to atmospheric absorption by water.

We now turn to the difference between the two measurements, which directly reflects the losses due to propagation through the four straight modules $(220 \mathrm{~mm}$ length). Below $550 \mathrm{GHz}$ the losses are too weak to be quantified reliably $(<1 \mathrm{~dB} / \mathrm{m})$. In the range $570-700 \mathrm{GHz}$ the average attenuation amounts to $3.4 \mathrm{~dB} / \mathrm{m}(6.4 \mathrm{~dB} / \mathrm{m}$ at 700 $750 \mathrm{GHz}$ ). The latter is still more than $40 \mathrm{~dB} / \mathrm{m}$ lower than a traditional WM-380 rectangular waveguide.

The data presented in Fig. 2 and Fig. 3 suggest that the weak attenuation observed at low frequencies is supported by the high $\mathrm{HE}_{11}$ mode purity. Higher frequencies exhibit lower mode purity, which is associated with measurable losses. Other factors, such as resonances between the converters (see raw data in Fig. 3), possible Bragg reflections at higher frequencies, and misalignment between the modules, have to be considered as well and require further analysis.

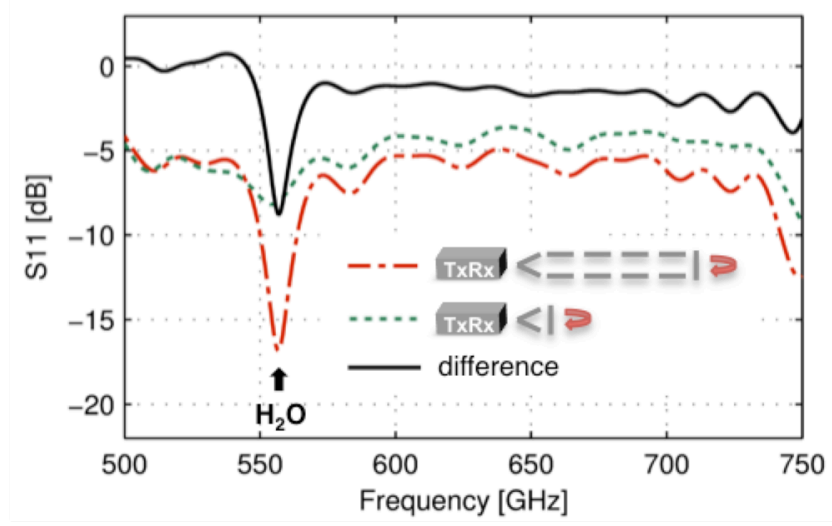

Fig. 4. $\mathrm{S}_{11}$ data (filtered, see Fig. 3). Configurations shown: Converter terminated by short (dashed line), converter and four 55 $\mathrm{mm}$ straight waveguide segments terminated by a short (dash-dotted line), difference between previous two datasets illustrating losses due to total round trip through a $440 \mathrm{~mm}$ long CCW (continuous line).

Additional measurements are performed in reflection $\left(\mathrm{S}_{11}\right)$ by terminating the two previous configurations with a short instead of a second converter (see sketches in Fig. 4). In this case the difference between the reference and the configuration with four added modules corresponds to a total transmission length of $440 \mathrm{~mm}$. As shown in Fig. 4, we still obtain close to zero losses for frequencies below $550 \mathrm{GHz}$ and an average attenuation of $3.1 \mathrm{~dB} / \mathrm{m}$ for 570 to $700 \mathrm{GHz}$, in line with the $\mathrm{S}_{21}$ data discussed above.

\section{Stability of Phase and Amplitude}

In particular test and measurement applications require high phase and amplitude stability and calibration capability. Fig. 5 shows long-term (4 hours) $\mathrm{S}_{21}$ measurements at $625 \mathrm{GHz}$ of (i) the two converters (face-to-face, $120 \mathrm{~mm}$ length) and (ii) a reference rectangular waveguide (1 inch WR-1.5). Both configurations yield very similar performance, dominated by
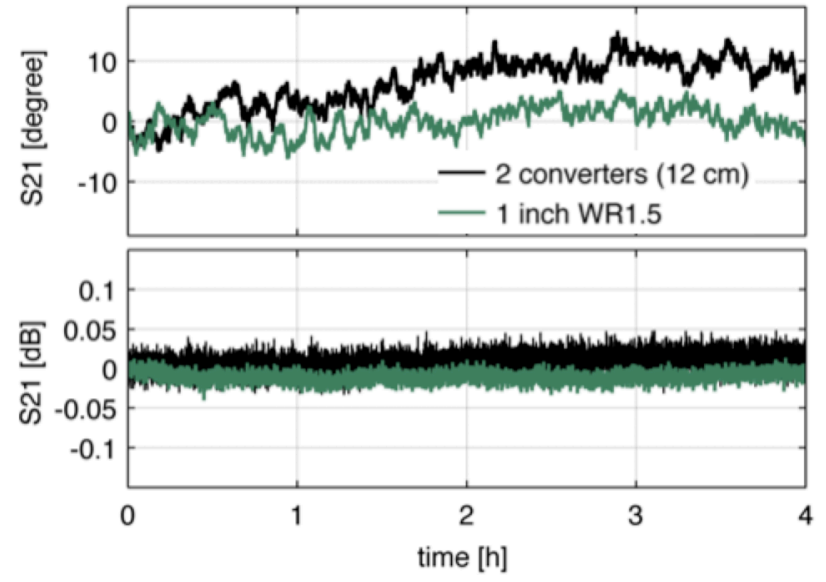

Fig. 5. Long-term stability of $\mathrm{S}_{21}$ at $625 \mathrm{GHz}$ (upper panel: phase, bottom panel: amplitude) for a pair of corrugated converters and a 1inch WR-1.5 fundamental rectangular waveguide segment.

noise and thermal drift due to the frequency extenders used. We observe a slightly increased noise and long-term drift for the corrugated converters, which can partly be attributed to the difference in length leading to increased thermal drift.

\section{SUMMARY}

A novel modular corrugated waveguide system for 500 to $750 \mathrm{GHz}$ based on the 'stacked rings' technology has been characterized in terms of losses and stability. Supported by an analysis of the propagating mode, the average waveguide losses were found to be more than four orders of magnitude lower than in conventional rectangular waveguides, opening the possibility for $\mathrm{THz}$ transmission over long distances. Furthermore, it was demonstrated that the long-term stability of the presented waveguide is on par with rectangular waveguides, enabling its use as a calibrated waveguide.

Thanks to its unprecedented performance and flexibility, this waveguide system is suitable for a wide range of $\mathrm{THz}$ applications. Several application platforms based on the presented components are currently under development. Among others, these will provide high-performance solutions for sub-THz to $\mathrm{THz}$ material characterization, on-wafer probing as well as gas spectroscopy.

\section{REFERENCES}

[1] "IEEE Approved Draft Standard for Rectangular Metallic Waveguides and Their Interfaces for Frequencies of $110 \mathrm{GHz}$ and Above. Part 1: Frequency Bands and Waveguide Dimensions", IEEE P17851D3 July 2012, pp. 1-20, 2012.

[2] J. L. Doane, "Propagation and mode coupling in corrugated and smooth-wall circular waveguides", in: Infrared and millimeter waves, Volume 13. Orlando, FL: Academic Press Inc., 1985.

[3] E. de Rijk, A. Macor, J.-Ph. Hogge, S. Alberti, and J.-P. Ansermet, "Note: Stacked rings for terahertz wave-guiding", Rev. Sci. Instrum., vol. 82, no. 6, p. 066102, 2011.

[4] E. d. Rijk, A. Macor, A. von Bieren, J.-P. Ansermet, B. Maffei, G. Pisano, and J. Hesler, "Modular set of corrugated wave- 
guiding components for applications from 500 to $750 \mathrm{GHz}$, in 38 th International Conference on Infrared, Millimeter, and Terahertz Waves (IRMMW-THz), pp. 1-2, 2013.

[5] B. Maffei, E. Gleeson, J. A. Murphy, and G. Pisano, "Study of corrugated Winston horns", in Astronomical Telescopes and Instrumentation, International Society for Optics and Photonics vol. 5498, pp. 812-817, 2004.

[6] S. K. Jawla, E. A. Nanni, M. A. Shapiro, P. P. Woskov, and R. J. Temkin, "Mode Content Determination of Terahertz Corrugated Waveguides Using Experimentally Measured Radiated Field Patterns," IEEE Trans. Plasma Sci., vol. 40, no. 6, pp. 1530-1537, 2012. 